\title{
ORBIT PERTURBATION ANALYSIS OF WEST FORD NEEDLES CLUSTERS
}

\author{
Bruce R. Bowman \\ Space Warfare Center, Analysis and Engineering Division \\ Schriever AFB, Colorado \\ William N. Barker, and William G. Schick \\ ITT Industries, Systems Division \\ Colorado Springs, Colorado
}

\begin{abstract}
The West Ford package placed in orbit in 1963 contained almost 500 million copper dipoles. Due to a delay in releasing the needles in orbit a number of clusters of needles formed. Analysis of 34 years of mean orbital elements of 20 different clusters has revealed perturbations of $10 \mathrm{~km}$ or more in the semimajor axis due to direct solar radiation pressure, atmospheric drag, and Earth albedo pressure. The orbits have perigee heights ranging from $1500 \mathrm{~km}$ to 3600 $\mathrm{km}$. A simplified Earth albedo model has been adopted to account for the long periodic perturbations observed in the semi-major axis. The periodic terms are a function of the sun and cluster nodal longitude angles. The atmospheric drag perturbation was modeled by extending the CIRA72 model atmosphere to heights above $2000 \mathrm{~km}$. Cluster area-to-mass ratios were determined from fitting direct solar radiation pressure coefficients over 25 to 30 year data spans. Twenty to thirty year semi-major axis fits produced mean atmospheric density factors for hydrogen and helium for each cluster. These factors show a very high correlation with average perigee height. The results show that average hydrogen concentrations are from 3 to 5 times greater than given by the CIRA72 model. Helium concentrations also are from 1.5 to 4 times greater than predicted for these heights.
\end{abstract}

\section{Introduction}

In May 1963 a package containing 500 million copper dipoles was placed into a near circular, nearly polar orbit at a mean altitude of $3650 \mathrm{~km}$. The purpose of this West Ford project was to establish an orbiting belt of dipole antennas, where messages could be sent coast to coast via the orbiting copper needles (Shapiro,
1964; Morrow, 1964; Waldron, 1964). The needles were each $1.78 \mathrm{~cm}$ long and $0.00178 \mathrm{~cm}$ in diameter. Approximately $25 \%$ to $40 \%$ of the dipoles were released as individual needles, with the rest released into orbit in approximately 100 clumps or clusters of needles. The large majority of these clusters are very small objects with radar cross sections less than $0.2 \mathrm{~m}^{2}$. The individual needles all decayed within 5 years, but only about $40 \%$ of the clusters have decayed. The remaining clusters in orbit have been tracked with difficulty over the last 37 years. Orbit fits indicate that these objects have large area-to-mass ratios which make them susceptible to large orbit perturbations from solar radiation pressure, atmospheric drag, and from the Earth's reflected solar radiation pressure (Earth albedo). The purpose of this paper is to present the results of the analysis of these perturbations on the orbits of the current needle clusters. Over 30 years of mean orbital element data has been analyzed to obtain long term orbit fits that accurately account for the perturbations effecting these clusters.

\section{Atmospheric Drag Perturbation}

Current atmospheric models employ tables that are for heights below 2000-2500 km. However, it has been shown from analysis (Afonso, 1985; Harwood, 1995) of LAGEOS data that atmospheric drag still has a measurable effect at $6000 \mathrm{~km}$. For the current analysis of the needles orbits the CIRA72 model atmosphere (COSPAR, 1972; Jacchia, 1970) was selected for use in the drag computations. This model integrates the diffusion equations as opposed to using lookup tables. Therefore, the CIRA72 model can be easily extended above the $2000 \mathrm{~km}$ height. 
The atmospheric drag is expressed by the drag force per unit of mass in the following form

$$
F=\frac{1}{2} \mathrm{C}_{\mathrm{D}} \rho \frac{A}{M} V_{S}^{2}
$$

$C_{D}$ is the drag coefficient (Cook,1966), $\rho$ is the atmospheric density, $\mathrm{A} / \mathrm{M}$ is the area-to-mass ratio, and $V_{S}$ denotes the satellite velocity with respect to the atmosphere. The drag coefficient depends upon the reflectivity of the satellite as well as the velocity of the satellite. For the velocity of the satellite greater than the thermal velocity $V_{T}$ of the gas particles, the drag coefficient can be expressed as (Afonso, 1985)

$$
\mathrm{C}_{\mathrm{D}}=\delta\left[2+\frac{4}{3}\left(\frac{V_{T}}{V_{S}}\right)^{2}-\frac{2}{15}\left(\frac{V_{T}}{V_{S}}\right)^{4}\right]
$$

$\delta$ is the accommodation coefficient representing the reflective properties of the satellite's surface. For a perfect absorber $\delta=1$, and for $\delta=2$ all incoming particles are reemitted in the opposite direction. For a spherical aluminum satellite Alfonso gives the following tables of values for $\mathrm{C}_{\mathrm{D}}$ and $\delta$ assuming a diffuse reemission process and a hyperthermal free flow (satellite velocity greater than thermal velocity).

\begin{tabular}{|l|c|}
\hline \multicolumn{2}{|l|}{ Accommodation Coef $\delta$} \\
\hline Hydrogen & 1.4 \\
\hline Helium & 1.3 \\
\hline Oxygen & 1.1 \\
\hline
\end{tabular}

Table 1. Accommodation coefficient $\delta$ for different molecular species.

\begin{tabular}{|c|cc|cc|cc|}
\hline \multicolumn{4}{c}{ Hydrogen } & \multicolumn{2}{c|}{ Helium } & \multicolumn{2}{c|}{ Oxygen } \\
\cline { 2 - 7 } $\mathbf{T}(\mathbf{K})$ & $\mathbf{V}_{\mathbf{T}} / \mathbf{V}_{\mathbf{S}}$ & $\mathbf{C}_{\mathbf{D}}$ & $\mathbf{V}_{\mathbf{T}} / \mathbf{V}_{\mathbf{S}}$ & $\mathbf{C}_{\mathbf{D}}$ & $\mathbf{V}_{\mathbf{T}} / \mathbf{V}_{\mathbf{S}}$ & $\mathbf{C}_{\mathbf{D}}$ \\
\hline 800 & 0.72 & 3.71 & 0.36 & 2.82 & 0.18 & 2.25 \\
1200 & 0.88 & 4.14 & 0.44 & 2.93 & 0.22 & 2.27 \\
2000 & 1.14 & 4.90 & 0.57 & 3.15 & 0.29 & 2.32 \\
\hline
\end{tabular}

Table 2. Drag coefficient $\mathrm{C}_{\mathrm{D}}$ and relative thermal velocity $V_{T} / V_{S}$ as a function of atmospheric temperature and molecular species.
For a low circular satellite, $\frac{V_{T}}{V_{S}} \sim 0.15$, the heavier species dominate $(\delta \sim 1.1)$. For this case the typical drag coefficient of 2.25 is used for drag computations. However, for higher altitude orbits the $C_{D}$ value increases. For a circular orbit at $3500 \mathrm{~km} \frac{V_{T}}{V_{S}} \sim 0.72$. At this height hydrogen dominates during periods of low solar activity $\left(\mathrm{T} \sim 600^{\circ} \mathrm{K}\right)$, while helium dominates during periods of high solar activity $\left(\mathrm{T} \sim 1500^{\circ} \mathrm{K}\right)$. Thus, the $C_{D}$ value can change from less than 3 to over 4 depending upon the exospheric temperature.

The drag analysis for this paper consisted of using orbit-averaged perturbation equations derived by KingHele (1964). The equations for the drag perturbation are based on an atmospheric density value obtained at approximately $1 / 2$ scale height, $H_{S}$, above the perigee height. With this method an error of up to $25 \%$ in scale height will result in an error less than $1.5 \%$ in the drag perturbation. The drag equation is a function of $\dot{a}, \rho$, $\mathrm{A} / \mathrm{M}, \mathrm{C}_{\mathrm{D}}$, and other orbital elements, where $\dot{a}$ is the change in semi-major axis with respect to time, and $\rho$ is the atmospheric density at $\sim 1 / 2 \mathrm{H}_{\mathrm{S}}$ above perigee. The equation can be represented as

$$
\dot{a}=\mathrm{C}_{\mathrm{D}} \frac{\mathrm{A}}{\mathrm{M}} \rho f\left(\mathrm{H}_{\mathrm{S}}, \text { orbital elements }\right)
$$

where $f$ represents a function of scale height and the orbit. From the previous $\mathrm{C}_{\mathrm{D}}$ discussion the breakdown of $\delta$ into density for each species is important. For the height range of $2000 \mathrm{~km}$ to $4000 \mathrm{~km}$ the main density constituent varies between $\mathrm{H}$ and $\mathrm{He}$ depending upon the exospheric temperature. Thus, equation (3) needs to be modified to account for the variable $C_{D}$ value. The resultant equation is

$$
\dot{a}=\sum_{i=1}^{6}\left\{\mathrm{C}_{\mathrm{D}_{i}} \rho_{i}\right\} \frac{\mathrm{A}}{\mathrm{M}} f\left(\mathrm{H}_{\mathrm{S}}, \text { orbital elements }\right)
$$

The density $\rho_{i}$ for each species $\mathrm{H}, \mathrm{He}, \mathrm{O}, \mathrm{N}_{2}, \mathrm{Ar}$, and $\mathrm{O}_{2}$ is obtained from the CIRA72 model at each integration step, and the drag coefficient is computed for each species. The sum of the products is then used to compute the drag perturbation. 


\section{Earth Albedo Perturbation}

Modeling the Earth albedo can be a very complex task. The Earth-reflected radiation pressure is not uniform but shows variations in space and time dependent upon surface conditions (i.e. snow, ice, water, desert, forest) and cloud cover (Volrouhlicky, 1993-4). The albedo effect can change by over $400 \%$ over latitude from pole to equator, and also can exhibit a strong variation from season to season. A number of papers have been written about modeling the Earth's albedo in the analysis of LAGEOS data (Anselmo, 1983; Prior, 1971; Volrouhlicky, 1993). One of the purposes of the current analysis is to model the long term albedo effects with orbit-averaged perturbation equations. Anselmo (1983) has developed perturbation equations on the semi-major axis accounting for the significantly different albedo of the Northern and
Southern hemispheres, and the strong seasonal dependencies. Three albedo coefficients A, B, D, and a phase angle $\alpha$ must be determined for each satellite. For the needle's nearly polar orbits $\left(l \sim 87^{\circ}\right)$ the orbit node regresses at only $0.1 \mathrm{deg} / \mathrm{day}$, which produces a large long term 3600 day periodic in $a$ represented by the A coefficient. The radial accelerations in $a$ are a function of $e$ which produces very long term components due to the direct solar radiation pressure perturbation on the orbit eccentricity. The following table lists the significant albedo periodic terms for a typical needle cluster. The arguments of the in-track albedo acceleration depend upon the longitude of the sun, $\lambda_{\square}$, and the right ascension of the orbit node, $\Omega$, while the radial acceleration also depends upon the orbit argument of perigee, $\omega$.

\begin{tabular}{|c|c|c|c|c|}
\hline Argument & Acceleration & Coefficient & Period & Amplitude \\
\hline & & & (Days) & $\Delta \mathrm{a}(\mathrm{m})$ \\
\hline $2 \lambda-\Omega$ & & & & \\
\hline$\Omega$ & In-Track & $\mathrm{A}$ & 173 & 33 \\
\hline$\lambda-\Omega$ & In-Track & $\mathrm{A}$ & 3600 & 1630 \\
\hline & & $\mathrm{B}$ & 332 & 280 \\
\hline$\lambda+\omega+\Omega$ & Radial & $\mathrm{D}$ & 2680 & 30 \\
\hline$\lambda+\omega-\Omega$ & Radial & $\mathrm{D}$ & 5490 & 1650 \\
\hline$\lambda+\omega$ & Radial & $\mathrm{D}$ & 10465 & 160 \\
\hline
\end{tabular}

Table 3. Periodic albedo perturbation accelerations on $a$ with amplitudes.

\section{Long Term Orbit Fit Results}

Least squares long term fits of the semi-major axis were obtained for over 20 needle cluster orbits. To obtain good solutions and account for the 10 to 15 year periodic albedo terms it was necessary to use at least 25 years of data. The long data spans that were available insured that at least 2 periods were available for solution of the A coefficient and the D coefficient (except for the $\lambda+\omega$ component).

The semi-major axis was integrated over 34 years using a semi-analytical integrator with a step size of 2 days. Orbit-averaged equations for $\dot{a}$ were used for direct solar radiation pressure (Koskela, 1962), atmospheric drag (King-Hele, 1964), and albedo (Anselmo, 1983). There are no long periodic or secular perturbations in $a$ from gravitational lunar-solar perturbations. To keep the perturbations on $a$ as close as possible to the real orbit perturbations the rest of the orbital elements were constrained to the values of the real mean elements over the 34 year span. This method avoids major non-linear variations in $a$, and allows good convergence in the coefficient solutions. The solution parameters included density correction factors for $\mathrm{H}$ and $\mathrm{He}$, a direct solar radiation pressure coefficient, the initial semi-major axis value, and the albedo coefficients. The drag $C_{D}$ value was computed as previously discussed. The $\mathrm{A} / \mathrm{M}$ term was obtained from using the solution of the direct solar radiation pressure coefficient, $\gamma \mathrm{A} / \mathrm{M}$, and a typical reflectivity coefficient of 1.05 (Harwood, 1995) for $\gamma$. The density factors for $\mathrm{H}$ and $\mathrm{He}$ were then obtained directly from the orbit-averaged drag equations. 
Figure 1 shows the perturbations computed using the orbit fitted coefficients of satellite 02377 . The drag perturbation is approximately $10 \mathrm{~km}$ over the data span, while the direct solar radiation pressure and albedo perturbations account for $8 \mathrm{~km}$ and $4 \mathrm{~km}$ respectively. The orbit height is approximately $3500 \mathrm{~km}$, so it is surprising to see the large magnitude of the

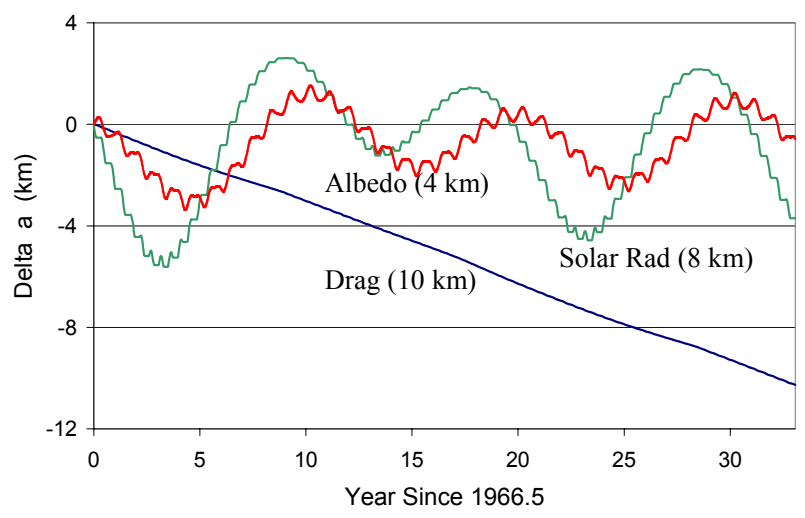

Fig. 1 Perturbations of the semi-major axis of satellite 02377

perturbations. However, this is easily explained by the large A/M ratio of $1.0 \mathrm{~m}^{2} / \mathrm{kg}$ that was obtained for this needle cluster. It was found that most of the other needle clusters had large $\mathrm{A} / \mathrm{M}$ ratios of at least 0.5 $\mathrm{m}^{2} / \mathrm{kg}$, some larger than $2.0 \mathrm{~m}^{2} / \mathrm{kg}$.

Figure 2 shows the residuals from fitting the NORAD mean $a$ values using 34 years of Satellite 02377 data. The standard deviation is $63 \mathrm{~m}$, showing that over $99 \%$ of the total $16 \mathrm{~km}$ perturbation in $a$ was accounted for. This demonstrates the validity of the orbit-averaged albedo model and the variable $C_{D}$ drag coefficient model. The orbit fits for the other satellites showed the same high improvement.

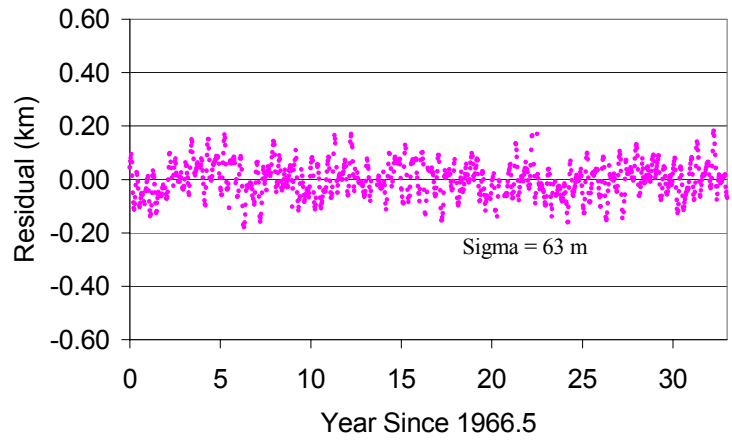

Fig. 2 Residuals in semi-major axis for satellite 02377 fit.
Table 4 lists the values obtained for the other orbit fits. The data span used for the fits are listed as $\Delta \mathrm{T}$ in years, and the total change in the semi-major axis is listed as $\Delta a$. The $\mathrm{A} / \mathrm{M}$ ratio was obtained from the fitted $\gamma \mathrm{A} / \mathrm{M}$ solar radiation pressure coefficient using $\gamma=1.05$. The standard deviation of each fit is given by the sigma value. The sigma values are all less than $1 \%$ of the total change in $a$, which shows the perturbation models are very good at fitting the mean $a$ values.

\begin{tabular}{|c|c|c|c|c|}
\hline NORAD & $\Delta \mathrm{T}$ & $\Delta a$ & $\mathbf{A} / \mathbf{M}$ & Sigma \\
\hline Satellite & Years & $\mathrm{Km}$ & $\mathrm{m}^{2} / \mathrm{kg}$ & $\mathrm{Km}$ \\
\hline & & & & \\
\hline 2359 & 31 & 27 & 1.52 & 0.095 \\
\hline 2360 & 32 & 79 & 2.06 & 0.279 \\
\hline 2361 & 29 & 6 & 0.73 & 0.037 \\
\hline 2362 & 29 & 54 & 1.32 & 0.122 \\
\hline 2363 & 21 & 76 & 1.52 & 0.074 \\
\hline 2364 & 29 & 8 & 0.75 & 0.057 \\
\hline 2367 & 30 & 88 & 1.53 & 0.261 \\
\hline 2374 & 20 & 84 & 1.70 & 0.191 \\
\hline 2375 & 33 & 55 & 1.19 & 0.208 \\
\hline 2377 & 33 & 16 & 0.97 & 0.063 \\
\hline 2378 & 29 & 10 & 0.58 & 0.046 \\
\hline 2379 & 28 & 37 & 1.28 & 0.120 \\
\hline 2380 & 29 & 20 & 1.17 & 0.075 \\
\hline 2431 & 34 & 19 & 0.95 & 0.088 \\
\hline 2496 & 25 & 59 & 1.84 & 0.295 \\
\hline 2530 & 33 & 22 & 1.20 & 0.130 \\
\hline 2796 & 28 & 23 & 1.02 & 0.110 \\
\hline 3235 & 26 & 79 & 1.89 & 0.257 \\
\hline 3257 & 29 & 14 & 0.98 & 0.065 \\
\hline 18962 & 19 & 109 & 1.53 & 0.267 \\
\hline 19002 & 18 & 75 & 2.00 & 0.206 \\
\hline & & & & \\
\hline
\end{tabular}

Table 4. Needle clusters used in the perturbation analysis.

No significant correlation was found between any two of the albedo coefficients. The only significant correlation between any albedo coefficient and the solar radiation pressure coefficient was for the radial albedo acceleration component. This is due to the radial albedo acceleration being a direct function of the orbital eccentricity, which is directly influenced by the solar radiation pressure perturbation. The non-correlation among the albedo coefficients demonstrates the complexity in using the three coefficients (A, B, and D) to obtain a close fit to the semi-major axis histories. 


\section{Validation of $\mathrm{A} / \mathrm{M}$ Values}

The $\mathrm{A} / \mathrm{M}$ ratios obtained from the direct solar radiation pressure coefficients required validation. Four of the needle clusters had their perigee heights drop below $1000 \mathrm{~km}$ for several years. Prior to this time the perigee heights were above $2000 \mathrm{~km}$ for 20 or more years. Figure 3 shows a plot of the perigee height of one of these four satellites.

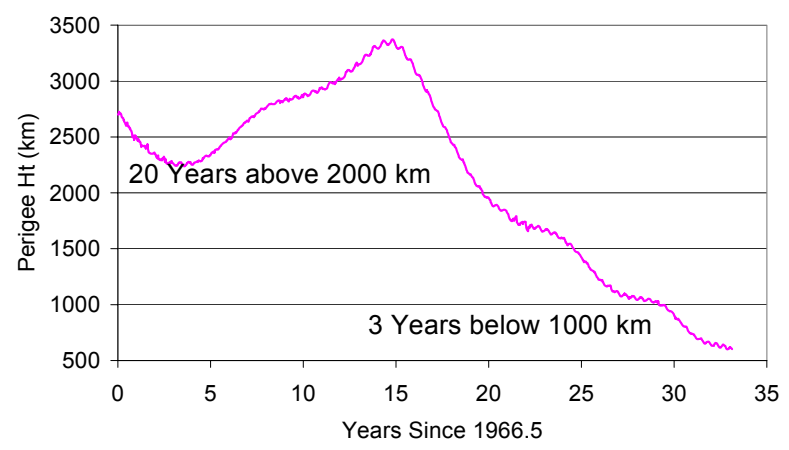

Fig. 3 Perigee height of satellite 02363 .

Solar radiation pressure coefficients were determined for the four satellites when the perigee heights were above $2000 \mathrm{~km}$. A reflectivity coefficient $\gamma$ of 1.05 was used to compute the $\mathrm{A} / \mathrm{M}$ values from the $\gamma \mathrm{A} / \mathrm{M}$ coefficient. For the time when perigee height was below $1000 \mathrm{~km}$ a Bterm $\left(\mathrm{C}_{\mathrm{D}} \mathrm{A} / \mathrm{M}\right)$ value was computed from the drag perturbation. These computations assumed the atmospheric density from the CIRA72 model was correct to within 15-20\%. The semi-major axis and Bterm were fit using data spans of 50 to 100 days during the several year span. The Bterms were averaged for each satellite during the time perigee height was below $1000 \mathrm{~km}$, and an average drag $\mathrm{A} / \mathrm{M}$ value was obtained using a $\mathrm{C}_{\mathrm{D}}$ value of 2.25 . Figure 4 shows the ratio of the solar radiation $\mathrm{A} / \mathrm{M}$

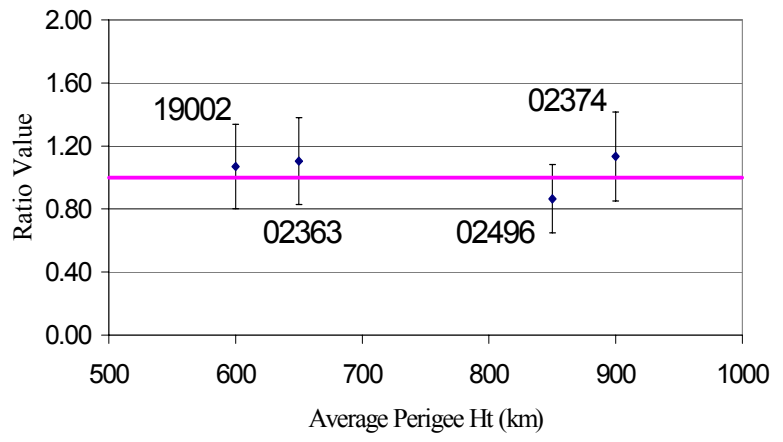

Fig. 4 Ratio of solar radiation pressure $\mathrm{A} / \mathrm{M}$ value to atmospheric drag $\mathrm{A} / \mathrm{M}$ at low perigee heights for 4 needle clusters. value to the low perigee height drag $\mathrm{A} / \mathrm{M}$ value. Also shown is the standard deviation (error bars) due to the Bterm averaging. The standard deviation (1 sigma) is about $25 \%$ of the $\mathrm{A} / \mathrm{M}$ values. The results are very good, showing that all the ratios fall within 1 sigma of the 1.00 value. This verifies that the $\mathrm{A} / \mathrm{M}$ values obtained from the solar radiation pressure fitted coefficients are equivalent to the $\mathrm{A} / \mathrm{M}$ values obtained from the drag perturbations at low altitudes. This is an extremely important result because it states that atmospheric density values can be accurately obtained at high altitudes when using the solar radiation pressure $\mathrm{A} / \mathrm{M}$ values.

\section{Atmospheric Density Results}

To solve for long term albedo and solar radiation pressure coefficients it was necessary to also solve for long term density factors to account for the drag perturbation on the semi-major axis. As a result the solutions for each satellite included a 20 to 30 year average $\mathrm{H}$ and $\mathrm{He}$ density factor that was applied to the CIRA72 model at each integration step. These factors are averages over 2 to 311 -year solar cycles and over perigee height variations of $\pm 500 \mathrm{~km}$ from an average value. These density factors are shown in Figures 5 and 6 for $\mathrm{H}$ and $\mathrm{He}$. The factors are plotted as a function of the minimum perigee height for each satellite during the entire 20-30 year data span (neglecting the times of low perigee height for the four satellites mentioned above). The consistency of the factor values shown in the figures is remarkable given the averaging over perigee heights and solar cycles. The $\mathrm{H}$ factor shows an offset

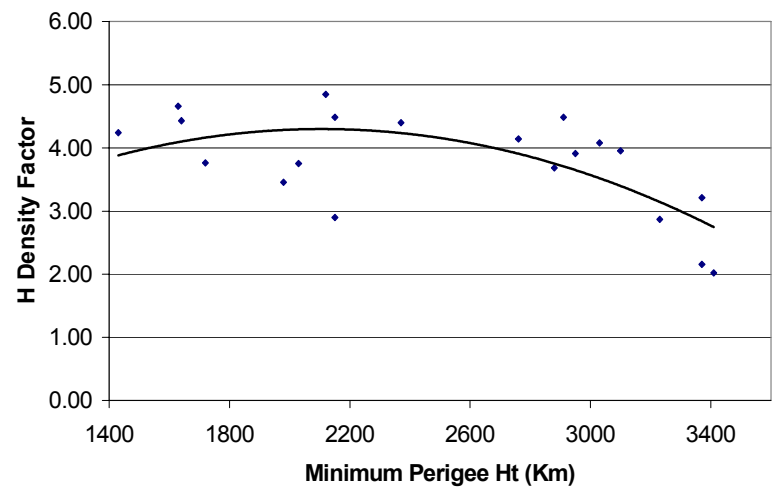

Fig. 5 Long term 20-30 year average hydrogen factors for needle clusters for different minimum perigee heights.

of between 3 and 5, indicating that the CIRA72 model $\mathrm{H}$ values are too low above $2000 \mathrm{~km}$. This is in good agreement with the Pageos and Dash 2 results (Prior, 1971; Rousseau, 1973) where the density was found to be a factor of 3 higher than predicted by the US Standard Atmospheric Supplements (1966). 


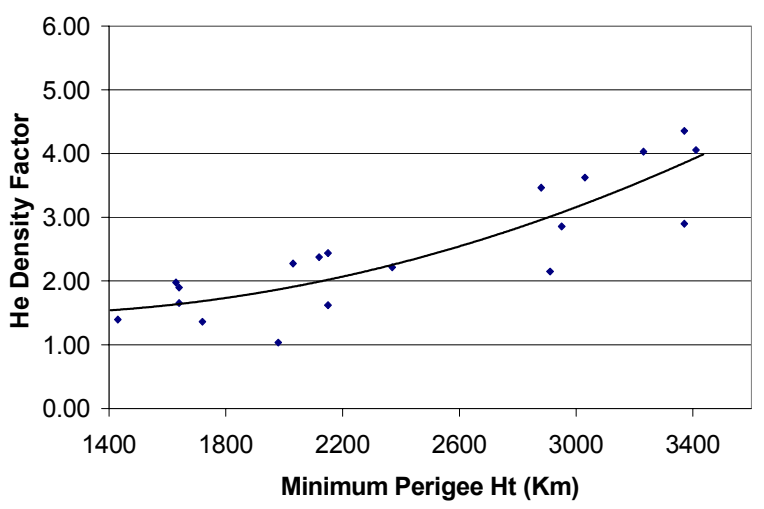

Fig. 6 Long term 20-30 year average helium density factors for different needle cluster minimum perigee heights.

The next step in the continuing analysis will be to compute time and height dependent $\mathrm{H}$ and He factors. This will be done by using $\mathrm{A} / \mathrm{M}$ values computed from the solar radiation pressure coefficients, and solving for the $\mathrm{H}$ and $\mathrm{He}$ factors using 1 to 2 year data spans.

\section{Conclusion}

The analysis has demonstrated the validity of using orbit-averaged equations to represent the long term Earth albedo perturbations. Long term least squares fits of mean element semi-major axis values can produce accurate coefficients for atmospheric drag, direct solar radiation pressure, and Earth albedo perturbations.

The long-term fitted solar radiation pressure coefficient can be accurately used to compute the A/M value of each satellite, which in turn can be used to compute atmospheric density values at high altitudes.

A variable drag coefficient model is required to obtain accurate density values for different molecular species. For high altitudes the model has been successfully used to obtain long term mean $\mathrm{H}$ and $\mathrm{He}$ density values.

\section{References}

Afonso, G., F. Barlier, C. Berger, F. Mignard, and J. J. Walch, Reassessment of the Charge and Neutral Drag of LAGEOS and its Geophysical Implications, Jour. Geophys Res., 90, 9381, 1985.

Anselmo, L., P. Farinella, A. Milani, and A. M. Nobili, Effects of the Earth-reflected Sunlight on the Orbit of the LAGEOS Satellite, Astron. and Astroph., 117, 3, 1983.

Cook, G. E., Drag Coefficients of Spherical Satellites, Ann. Geophys., 22, 53, 1966.

COSPAR International Reference Atmosphere 1972, Compiled by the members of COSPAR Working Group 4, Akademie-Verlag, Berlin, 1972.

Harwood, N. M., and G. G. Swinerd, Long-Periodic and Secular Perturbations to the Orbits of Explorer 19 and LAGEOS Due to Direct Solar Radiation Pressure, Cel. Mech. And Dynam. Astron., 62, 81, 1995.

Jacchia, L. G., New Static Models of the Thermosphere and Exosphere with Empirical Temperature Profiles, Smithson. Astrophys. Special Report 313, 1970.

King-Hele, D., Theory of Satellite Orbits in an Atmosphere, Butterworths, London, 1964.

Koskela, P. E., Orbital Effects of Solar Radiation Pressure on an Earth Satellite, Journal of Astronautical Sciences, 9, 71, 1962.

Morrow, W. E., and T. F. Rogers, The West Ford Experiment - An Introduction to this Issue, Proceedings of the IEEE, 52, 461, 1964.

Prior, E. J., Observed Effects of Earth-Reflected Radiation and Hydrogen Drag on the Orbital Accelerations of Balloon Satellites, Symposium on the Use of Artificial Satellites for Geodesy, Washington, D.C., 1971.

Rousseau, M., Densities Deduced from Perturbations at High Altitudes, Planet. Space Sci., 21, 1705, 1973.

Shapiro, I. I., H. M. Jones, and C. W. Perkins, Orbital Properties of the West Ford Dipole Belt, Proceedings of the IEEE, 52, 469, 1964.

Vokrouhlicky, D., Albedo Perturbation Models: General Formalism and Applications to LAGEOS, Cel. Mech. And Dynam. Astron. 57, 225, 1993.

Vokrouhlicky, D., P. Farinella, and D. Lucchesi, LongPeriodic Albedo Perturbations on LAGEOS, Astron. Astrophys., 280, 282, 1993.

Vokrouhlicky, D., P. Farinella, and F. Mignard, Solar Radiation Pressure Perturbations for Earth Satellites, III. Global Atmospheric Phenomena and the Albedo Effect, Astron. Astrophys, 290, 324, 1994.

Waldron, P., D. C. MacLellan, and M. C. Crocker, The West Ford Payload, Proceedings of the IEEE, 52, $571,1964$. 